\title{
Aspectos Fisiológicos e Biomecânicos da Produção de Força Podem ser Usados no Controle do Treinamento de Remadores de Elite
}

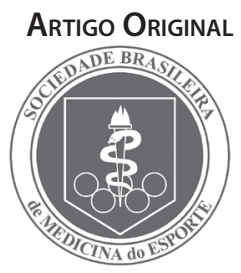

\author{
Physiological and Biomechanical Aspects of Force Production Can be \\ Used in the Training Control of Elite Rowers
}

Rafael Reimann Baptista ${ }^{1,2}$

Giovani dos Santos Cunha'

Alvaro Reischak de Oliveira

1. Universidade Federal do Rio Grande do Sul - Escola de Educação Física - LAPEX - Porto Alegre/RS 2. Universidade Luterana do Brasil (ULBRA) - Campus Gravataí - Curso de Educação Física

\section{Endereço para correspondência:} Alvaro Reischak de Oliveira Endereço: Rua Felizardo, no. 750, ESEF/UFRGS - Laboratório de

Pesquisa do Exercício

CEP 90690-200

Porto Alegre - RS - Brasil

E-mail: aroliveira@esef.ufrgs.br

Submetido em 15/01/2008

Versão final recebida em 23/02/2008 Aceito em 04/04/2008

\begin{abstract}
RESUMO
Atualmente, a ciência do esporte requer abordagens que combinem o estudo de aspectos biomecânicos e fisiológicos para o correto entendimento dos fenômenos relacionados com o desempenho humano. 0 objetivo deste estudo foi investigar as diferenças nas respostas fisiológicas e biomecânicas entre as diferentes curvas força $x$ tempo de remadores. A amostra foi composta de 15 remadores (23,6 \pm 5,4 anos), submetidos a um teste máximo em remoergômetro. As respostas fisiológicas $\left(\mathrm{VO}_{2}, V \mathrm{VO}_{2}, \mathrm{VE}\right.$, RER MET, lactato e $\left.F C\right)$ e biomecânicas (pico de força, potência e impulso) foram mensuradas no estágio de limiar de lactato, bem como no estágio máximo alcançado pelos remadores durante o teste em remoergômetro. Os remadores foram classificados em dois grupos de acordo com a localização do pico de força em suas curvas força $x$ tempo: pico de força na primeira metade da curva (stroke) ou na segunda metade da curva (bow). Não foram encontradas diferenças significativas entre os parâmetros analisados nos dois grupos no estágio de limiar de lactato. Dos 12 remadores que apresentaram perfil bow no estágio de limiar de lactato, 41,7\% transitaram para o perfil stroke, enquanto 58,3\% mantiveram o perfil original no estágio máximo, os quais apresentaram maior produção de potência $(p<0,05)$. Os resultados sugerem que treinadores interessados em prolongar o tempo de exercício devem procurar um perfil bow da curva força $x$ tempo e remadores com perfil stroke poderiam ser melhor adaptados a barcos rápidos.
\end{abstract}

Palavras-chave: fadiga, lactato, força muscular.

\section{ABSTRACT}

The sports science currently requires approaches that match the study of biomechanical and physiological aspects, for the correct understanding of the phenomena related to human performance. The aim of this study was to investigate the differences in biomechanical and physiological responses between different force-time curves in rowers. Fifteen rowers (23.6 $\pm 5.4 \mathrm{yrs})$, were submitted to maximal rowing ergometer. The biomechanical (peak force, power and impulse) and physiological $\left(\mathrm{VO}_{2}, \mathrm{VCO}_{2}, \mathrm{VE}, \mathrm{RER} \mathrm{MET}\right.$, lactate and HR) responses were measured in the lactate threshold stage as well as in the maximal stage reached by the rowers. The rowers were classified in two groups according to the localization of the peak force in their force-time curves: peak force in the first half of the curve (stroke) or in the second half of the curve (bow). There are no significant differences between the parameters measured in the two groups at lactate threshold stage of the rowing ergometer. Twelve rowers presented finish accentuated force curve in the lactate threshold; however, five (41.7\%) changed for a catch accentuated pattern, while seven (58.3\%) kept the original force-time profile in the maximal stage of the rowing ergometer. The rowers with a finish accentuated profile showed higher power production $(p<0.05)$. The results suggest that coaches interested in extending exercise time must seek a finish accentuated force-time profile. Catch accentuated rowers could be better adapted to faster boats.

Keywords: fatigue, lactate, muscle strength.

\section{INTRODUCÃO}

O remo é um esporte em que os atletas produzem grandes quantidades de força, algumas vezes o pico de força alcança valores de 1.000 a $1.500 \mathrm{~N}$ na largada da regata e, durante a prova, a velocidade é mantida em menor nível, com o pico de força na ordem de 500 a $700 N^{(1)}$. Além disso, o remo é um esporte que envolve um gesto esportivo complexo ${ }^{(2,3)}$. Assim, a compreensão dos aspectos fisiológicos e biomecânicos que influenciam a produção de força de remadores é um fator importante para aperfeiçoar o desempenho desses atletas( ${ }^{(4)}$
Muitos estudos mensuraram a curva de produção de força durante o tempo de sua aplicação na remada, analisando alguns parâmetros que poderiam interferir no desempenho dos remadores ${ }^{(5-7)}$. Existem evidências de que os remadores são consistentes no seu perfil de curva força x tempo e que alguns atletas são capazes de alterar esse padrão de produção de força com o treinamento(4,8).

Remadores que alcançam seu pico de força na primeira metade da curva força x tempo são chamados stroke, caracterizados por maiores concentrações de lactato e de fibras glicolíticas de ativação rápida no 
músculo deltóide do que remadores que apresentam o pico de força na segunda metade da curva força x tempo, chamados de bow, que apresentam menor concentração de lactato e mais fibras oxidativas de ativação lenta no mesmo músculo( ${ }^{(9)}$.

Atualmente, principalmente em se tratando de esportes de alto rendimento, abordagens unilaterais envolvendo apenas aspectos fisiológicos como a freqüência cardíaca, consumo de oxigênio, lactato, entre outros, ou apenas aspectos biomecânicos, como força, potência e torque, proporcionam uma contribuição limitada para a produção de conhecimento na área das ciências do esporte.

Entender as relações entre as variáveis fisiológicas e biomecânicas em um esporte ajuda a alcançar o máximo desempenho dos atletas. Dentre essas interações, as relações entre diferentes estratégias de produção de força e as respostas cronotrópicas, ventilatórias e metabólicas são extremamente importantes em um esporte como o remo.

Poucos estudos buscaram compreender os fenômenos fisiológicos e biomecânicos do remo dentro de uma perspectiva multidisciplinar. Assim, o principal objetivo deste artigo foi comparar as respostas fisiológicas de consumo de oxigênio, produção de dióxido de carbono, ventilação, taxa de troca respiratória, equivalente metabólico, lactato e freqüência cardíaca e as variáveis biomecânicas de força, potência e impulso entre remadores com perfil de curva força x tempo stroke e bow.

Nossa principal hipótese era a de que remadores com o perfil bow apresentassem predominância do metabolismo aeróbio durante o teste em remoergômetro, com menor acúmulo de lactato sanguíneo e maior tolerância ao exercício (tempo para alcançar a fadiga).

\section{MÉTODOS}

\section{Sujeitos}

A amostra foi composta de 15 remadores voluntários de um clube local de remo. Todos os sujeitos foram informados sobre os procedimentos e assinaram o termo de consentimento de acordo com a Declaração de Helsinque. O estudo foi aprovado pelo comitê de ética da Universidade Federal do Rio Grande do Sul. Todos os sujeitos treinavam utilizando a combinação barco específico de remo e remoergômetro, bem como treinamento de força e resistência. Os indivíduos eram heterogêneos em nível técnico, entretanto, possuíam em média 6,8 \pm 2,8 anos de experiência competitiva no esporte.

\section{Protocolo de teste}

Os remadores realizaram uma avaliação cineantropométrica composta por estatura, massa corporal e percentual de gordura. Todos os sujeitos realizaram um teste máximo em remoergômetro (Concept II, model C, Morrisville, VT, EUA). A carga inicial foi de 150 watts (W) e aumentada em 50W a cada três minutos, com 60 segundos de intervalo para a coleta de lactato sangüíneo e ajustes na aquisição dos dados referentes à força. Todos os atletas foram incentivados verbalmente para que alcançassem o máximo de desempenho; todos os atletas eram bem adaptados ao teste em remoergômetro.

Foram mensuradas variáveis fisiológicas como o consumo de oxigênio $\left(\mathrm{VO}_{2}\right)$, produção de dióxido de carbono $\left(\mathrm{VCO}_{2}\right)$, ventilação (VE), taxa de troca respiratória (RER) - razão entre $\mathrm{VCO}_{2} \mathrm{NO}_{2}$, equivalente metabólico (MET) - custo metabólico calculado através da energia fornecida pelo $\mathrm{O}_{2}$, lactato (La) e freqüência cardíaca (FC). As variáveis biomecânicas analisadas foram a força (F), potência (P) e impulso (I). Os dados ventilatórios foram coletados pelo método breath by breath através de um ergoespirômetro (CPX/D, Medical Graphics, St. Paul, MN, EUA). A FC foi mensurada através de um freqüencímetro (Polar Electro Oy, Kempele, Finlândia). As concentrações de lactato no plasma foram coletadas do lóbulo da orelha e determinadas por um analisador enzimático de lactato (Roche Bio-Electronics, Basiléia, Suiça).
A produção de força foi mensurada através de um transdutor de força juntamente com uma célula de carga (desenvolvida pelo Laboratório de Medições Mecânicas da UFRGS) acoplada entre a correia do remoergômetro e o pegador deste. A célula de carga foi calibrada em duas etapas: uma de aquisição e outra de análise. Na primeira etapa da calibração foi medida a ausência completa de produção de força pela célula de carga durante cinco segundos e após foi medida a produção de força por uma carga conhecida (1lb) durante cinco segundos, gerando um gráfico com diferentes sinais elétricos: zero, 1llb, 2lb, 3lb e 4lb. A segunda etapa consistia de uma análise pelo método FFT (fast Fourier transformation) da aquisição do sinal, filtragem do sinal e finalmente a conversão do sinal em newtons.

A potência produzida foi mensurada por um dispositivo do próprio remoergômetro ao longo de toda a duração dos estágios (três minutos cada estágio), enquanto o impulso era calculado a posteriori através da integral entre a relação força $x$ tempo por meio de um programa de computador para aquisição e análise de sinais biológicos (SAD32 - desenvolvido pela Faculdade de Engenharia da UFRGS).

\section{Análise estatística}

Os dados foram analisados no estágio de limiar de lactato, bem como no estágio máximo alcançado pelos atletas durante o teste máximo em remoergômetro (até a fadiga). O limiar de lactato foi identificado através do método Dmax, que interpola os valores de lactato com a potência produzida e sofre análise por inspeção visual do avaliador e confirmação por dois fisiologistas independentes ${ }^{(10)}$. Esse é um método de determinação individual do limiar anaeróbio que já foi utilizado pelo nosso grupo em estudo anterior, mostrando ser mais efetivo do que a determinação do limiar anaeróbio pelo método de concentração fixa de lactato sangüíneo de $4 \mathrm{mM}$, o qual superestima a intensidade de exercício em remadores ${ }^{(11)}$

A produção de $F$ foi medida conforme descrito anteriormente e usada para análise da curva força x tempo média dos remadores por meio do cálculo da duração da curva de força (tempo entre o início e o final da curva de força), a metade da curva (metade do tempo da curva de força) e o tempo em que ocorreu o pico de força (tempo do início da curva até o ponto onde ocorre o pico de força) usando um programa de computador específico (SAD32) para classificar os remadores como stroke ou bow (pico de força na primeira metade ou na segunda metade da curva força x tempo, respectivamente). A figura 1 apresenta esses parâmetros esquematicamente, sobrepondo várias curvas força x tempo de um mesmo remador. A figura 2 apresenta uma curva de força real de um remador após todo o tratamento do sinal.

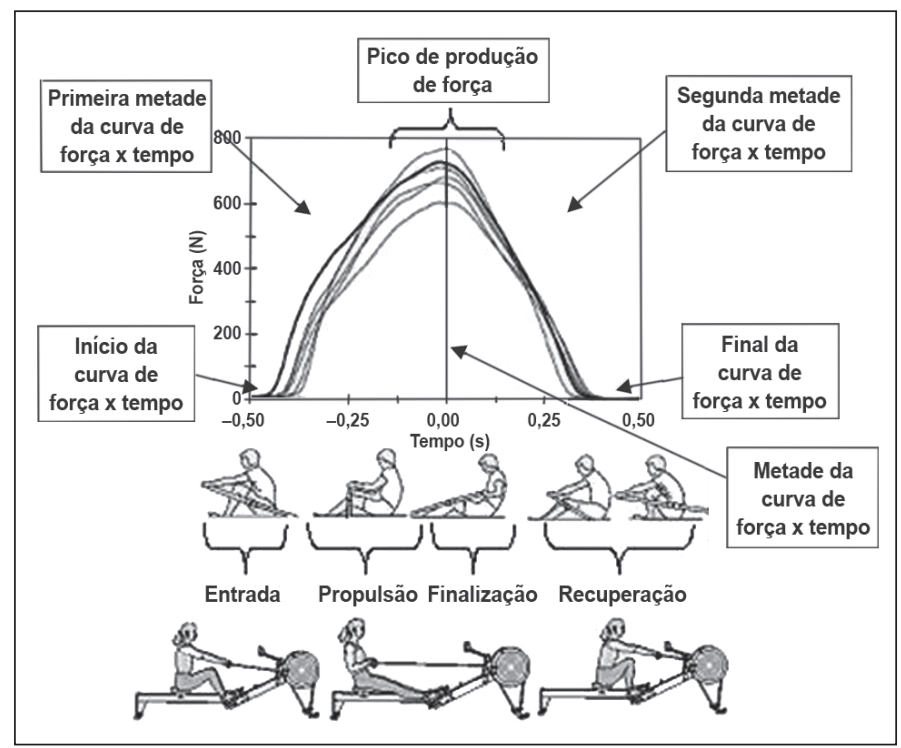

Figura 1. Descrição da curva força x tempo 


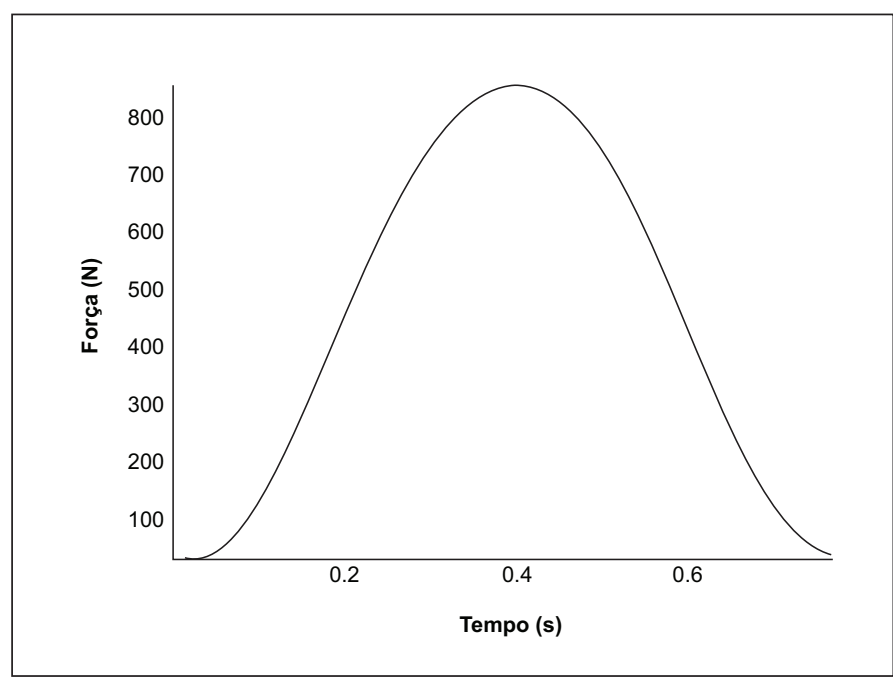

Figura 2. Representação da curva força x tempo após o tratamento do sinal

$\mathrm{O} \mathrm{VO}_{2}, \mathrm{MET}, \mathrm{VCO}_{2}, \mathrm{VE}$, RER, FC e I foram calculados para cada intensidade. Os valores de $\mathrm{P}$ e La foram utilizados como parâmetros de intensidade e coletados a cada estágio do teste e analisados posteriormente.

No tratamento estatístico, para comparar as variáveis fisiológicas e biomecânicas entre os grupos de remadores stroke e bow, tanto no limiar anaeróbio quanto no estágio máximo do teste em remoergômetro, foi utilizado o teste $t$ de Student para amostras independentes. $O$ teste do qui-quadrado de McNemar foi usado para avaliar as mudanças no comportamento da curva de força dos remadores entre os estágios de teste do limiar anaeróbio e máximo. O nível de significância adotado foi de $p<0,05$ e todo o tratamento estatístico foi realizado no programa SPSS 10.0 .

\section{RESULTADOS}

Algumas das características físicas e fisiológicas dos atletas são apresentadas na tabela 1.

Tabela 1. Características físicas e fisiológicas dos remadores

\begin{tabular}{c|c|c|c|c}
\hline $\begin{array}{c}\text { Idade } \\
(\text { anos })\end{array}$ & $\begin{array}{c}\text { Massa Corporal } \\
(\mathrm{kg})\end{array}$ & Estatura $(\mathrm{cm})$ & $\%$ de gordura & $\begin{array}{c}\mathrm{VO}_{2 \max } \\
\left(\mathrm{ml}^{\mathrm{kg}} \cdot \mathrm{kg}^{-1}\right)\end{array}$ \\
\hline $24,4 \pm 5,2$ & $83,5 \pm 7,1$ & $185,5 \pm 6,5$ & $13,6 \pm 1,6$ & $60,9 \pm 3,4$ \\
\hline
\end{tabular}

Valores representados por média \pm desvio-padrão

No estágio de limiar de lactato, foi possível identificar três remadores com perfil stroke e 12 remadores com perfil bow, não apresentando diferenças estatísticas nas respostas fisiológicas e biomecânicas. Verificamos que sete remadores com perfil bow (58,3\%) mantiveram o mesmo perfil da curva força $x$ tempo durante todo o teste em remoergômetro; entretanto, cinco remadores $(41,7 \%)$ alteraram seu padrão para o perfil stroke. No estágio máximo alcançado durante o teste em do remoergômetro, foram identificados oito remadores com perfil stroke e sete remadores com perfil bow. O grupo bow apresentou maior produção de potência máxima do que o grupo stroke $(p<0,05)$. Além disso, o grupo bow apresentou menores valores de FC e lactato sanguíneo do que o grupo stroke no estágio máximo de exercício, mas esses valores não foram estatisticamente significativos $(p>0,05)$. Não foram estabelecidas diferenças significativas em outras variáveis analisadas (tabela 2).
Tabela 2. Comparação das variáveis fisiológicas e biomecânicas no estágio de limiar de lactato e estágio máximo em remadores

\begin{tabular}{c|c|c|c|c}
\hline \multirow{2}{*}{} & \multicolumn{2}{|c|}{ Estágio limiar lactato } & \multicolumn{2}{c}{ Estágio máximo } \\
\cline { 2 - 5 } & $\begin{array}{c}\text { Stroke } \\
(\mathbf{n}=\mathbf{3})\end{array}$ & $\begin{array}{c}\text { Bow } \\
(\mathbf{n}=\mathbf{1 2})\end{array}$ & $\begin{array}{c}\text { Stroke } \\
(\mathbf{n}=\mathbf{8})\end{array}$ & $\begin{array}{c}\text { Bow } \\
(\mathbf{n}=\mathbf{7})\end{array}$ \\
\hline Massa Corporal $(\mathrm{kg})$ & $80,3 \pm 6,3$ & $84,3 \pm 7,4$ & $80,8 \pm 5,4$ & $86,5 \pm 8,2$ \\
\hline $\mathrm{FC}(\mathrm{bpm})$ & $148 \pm 16$ & $150 \pm 17$ & $186 \pm 8$ & $182 \pm 11$ \\
\hline $\mathrm{VO}_{2}\left(\mathrm{~m}^{\prime} \cdot \mathrm{kg}^{-1} \cdot \mathrm{min}^{-1}\right)$ & $39,43 \pm 3,02$ & $40,30 \pm 2,11$ & $60,65 \pm 3,94$ & $61,24 \pm 3,06$ \\
\hline $\mathrm{VO}_{2}\left(\mathrm{l} \cdot \mathrm{min}^{-1}\right)$ & $3,15 \pm 0,07$ & $3,39 \pm 0,30$ & $5,01 \pm 0,34$ & $5,16 \pm 0,48$ \\
\hline $\mathrm{MET}(\mathrm{METs})$ & $11,27 \pm 0,87$ & $11,51 \pm 0,60$ & $17,32 \pm 1,13$ & $17,50 \pm 0,89$ \\
\hline $\mathrm{VCO}{ }_{2}\left(\mathrm{I} \cdot \mathrm{min}^{-1}\right)$ & $2,72 \pm 0,96$ & $3,04 \pm 0,33$ & $4,42 \pm 1,81$ & $5,11 \pm 0,66$ \\
\hline $\mathrm{RER}$ & $0,88 \pm 0,02$ & $0,92 \pm 0,05$ & $1,47 \pm 0,08$ & $1,48 \pm 0,12$ \\
\hline $\mathrm{La}(\mathrm{mM})$ & $2,10 \pm 0,72$ & $2,87 \pm 1,08$ & $8,85 \pm 2,20$ & $6,77 \pm 1,76$ \\
\hline $\mathrm{P}(\mathrm{W})$ & $267 \pm 29$ & $275 \pm 34$ & $394 \pm 32$ & $436 \pm 38 *$ \\
\hline $\mathrm{F}(\mathrm{N})$ & $886 \pm 43$ & $830 \pm 64$ & $957 \pm 61$ & $901 \pm 76$ \\
\hline $\mathrm{I}\left(\mathrm{N} . \mathrm{s}^{-1}\right)$ & $395,29 \pm 18,02$ & $389,80 \pm 36,53$ & $354,52 \pm 30,77$ & $372,62 \pm 28,83$ \\
\hline
\end{tabular}

Valores apresentados por média \pm desvio-padrão $\left.{ }^{*} \mathrm{p}<0,05\right)$, freqüência cardíaca $(\mathrm{FC})$, consumo de oxigênio relativo $\left(\mathrm{ml}^{\mathrm{kg}} \mathrm{kg}^{-1} \cdot \mathrm{min}^{-1}\right)$, consumo de oxigênio absoluto (l. $\left.\mathrm{min}^{-1}\right)$, equivalente metabólico (MET), produção de dióxido de carbono $\left(\mathrm{VCO}_{2}\right)$, taxa de troca respiratória (RER), lactato (La), potência (P), pico de força (F) e impulso (I).

\section{DISCUSSÃO}

As principais descobertas deste estudo foram que alguns remadore, quando submetidos a um teste máximo em remoergômetro, alteram seu perfil da curva força $x$ tempo e que, remadores que mantiveram o perfil bow da curva força $x$ tempo apresentaram maiores valores de potência máxima. Para nosso conhecimento, somente o estudo de Roth et al..$^{(9)}$ comparou as características fisiológicas de remadores com perfil bow e stroke, principalmente focalizando as diferenças na composição de fibras musculares e lactato sangüíneo. Assim, poderia ser levaantada a hipótese de que remadores com diferentes padrões de curva força $x$ tempo poderiam apresentar diferentes respostas fisiológicas, como a predominância do metabolismo anaeróbio em remadores com perfil stroke e maior participação do metabolismo aeróbio em remadores com perfil bow.

Nossos resultados sugerem que perfil de produção de força apresentado pelo grupo bow é mais eficiente do que o do grupo stroke, o que vai ao encontro do estudo de Roth et al.(9), uma vez que os remadores bow produziram maior potência máxima (436 vs. 394W) do que os remadores stroke, enquanto a maioria dos parâmetros fisiológicos foi similar. O consumo de oxigênio foi praticamente o mesmo e as concentrações de lactato foram menores no grupo bow.

Além disso, a massa corporal que poderia explicar a maior potência máxima no grupo bow, devido ao seu impacto na produção de potência em remadores treinados principalmente quando avaliados em remoergômetro, não se mostrou significativamente diferente entre os grupos (tabela 2).

O único parâmetro fisiológico maior no grupo bow, ainda que sem diferença estatisticamente significativa, foi a produção de dióxido de carbono $\left(\mathrm{VCO}_{2}\right)$. Poderia essa variável estar relacionada com maior contribuição do metabolismo anaeróbio? Essa interpretação não seria necessariamente uma contradição aos menores valores de lactato no grupo bow, pois o limiar anaeróbico difere entre os indivíduos, e as concentrações de lactato sangüíneo são menores quanto maior a aptidão aeróbica dos atletas. 
Nossos resultados sugerem que no grupo estudado as respostas fisiológicas são semelhantes. Entretanto, a biomecânica do remo mostrou que remadores com perfil bow podem suportar o exercício em remoergômetro por um período maior de tempo do que os remadores com perfil stroke, verificado pela maior potência alcançada. Estudos anteriores relataram que alta produção de potência é positivamente correlacionada com o desempenho durante um teste de 2.000 me$\operatorname{tros}^{(12-14)}$, teste tipicamente utilizado para predizer o desempenho em provas de remo.

Hill(8) verificou que, apesar de os remadores apresentarem padrões individuais na produção de força, os que treinam juntos durante um longo período de tempo são capazes de sincronizar seus movimentos e assim modificar seus padrões de curva de força. Adicionalmente, alguns autores demonstraram alta reprodutibilidade nas estratégias de remada entre atletas, o que suporta a idéia de que remadores são capazes de reproduzir seus padrões de curva força $x$ tempo ${ }^{(4)}$. Verificamos que alguns remadores podem realmente manter seus padrões de curva força x tempo durante um teste em remoergômetro, enquanto outros apresentaram alterações ao longo do teste de exercício. Provavelmente, a causa dessas alterações foi a fadiga pelo aumento da intensidade do exercício durante a progressão dos estágios do teste em remoergômetro, que pode conduzir a aumento de metabólitos acumulados no músculo, causando limitada produção de força e manutenção da potência(15). Da mesma forma, o aumento da intensidade induz maior recrutamento de fibras musculares, especialmente fibras glicolíticas do tipo Ilb, as quais são altamente fatigáveis ${ }^{(16)}$.

Nossos resultados estão de acordo com estudos anteriores, confirmados pela maior potência máxima alcançada por remadores com perfil bow e pela modificação da curva força $x$ tempo apresentada por remadores que reduziram seu tempo para alcançar seu pico de força, alterando assim seu perfil de bow para stroke durante um teste máximo em remoergômetro.

Tendo em vista que maior produção de potência durante o teste máximo em remoergômetro é relacionada com maior duração do teste, treinadores interessados em melhorar o desempenho de seus remadores poderiam adaptar seus atletas a reproduzirem o perfil bow da curva força x tempo. Esse perfil apresenta-se como mais eficiente, aumentando a probabilidade de melhor desempenho em provas. Não obstante, mais estudos são necessários para confirmar se remadores com perfil bow realmente apresentam melhor desempenho em provas de 2.000 metros.

Nesta óptica, novos estudos analisando o consumo de oxigênio de remadores durante um exercício em remoergômetro, alternando os padrões de curva de força com feedback visual, poderão esclarecer se remadores com perfil bow são mais econômicos do que remadores com perfil stroke.

Adicionalmente, a monitoração da curva força x tempo pode ser um critério para estabelecer a tripulação dos barcos de competição. Grandes barcos, com mais remadores que alcançam maiores velocidades e onde a aplicação da força deve ser mais rápida, parecem ser mais adequados para remadores com perfil stroke. Barcos menores com poucos ou apenas um remador, onde o tempo de aplicação de força por remada é maior, parecem ser mais adequados para remadores com perfil bow.

A combinação dos resultados indica que remadores que mantiveram o perfil bow da curva força $x$ tempo alcançam maiores valores de potência máxima do que os que modificaram o formato de sua curva força $x$ tempo. Essa mudança parece não obedecer a nenhum padrão específico, contrariando estudos anteriores que demonstraram manutenção da curva força x tempo em remadores bem treinados ${ }^{(8)}$. Entretanto, variações na voga (freqüência de remada) utilizada pelos remadores durante os testes podem ter influenciado os resultados, uma vez que a velocidade de movimento influencia a capacidade de produção de força. Assim, devemos considerar esse aspecto como uma limitação do estudo.

\section{CONCLUSÕES}

Em nosso estudo, os remadores stroke e bow não apresentaram diferenças significativas nas variáveis fisiológicas e biomecânicas, com exceção da potência máxima produzida. Dessa forma, nossa hipótese de que os remadores bow apresentariam perfil fisiológico mais aeróbio foi negada. Verificamos ainda que os remadores apresentaram alteração de sua estratégia de produção de força durante um teste máximo em remoergômetro, em que os remadores com perfil bow da curva força x tempo foram capazes de produzir maior potência do que remadores stroke.

\section{AGRADECIMENTOS}

Ao CNPq, CENESP e LAPEX pelo auxílio financeiro. A Gabriel Figueiredo e Letícia de Oliveira pelo auxílio nas coletas de dados. Ao treinador Ricardo Contieri pela liberação dos atletas para participação neste estudo. Aos diversos pesquisadores que contribuíram com sugestões e comentários, especialmente Dr. Holger Hill.

Todos os autores declararam não haver qualquer potencial conflito de interesses referente a este artigo.

\section{REFERÊNCIAS BIBLIOGRÁFICAS}

1. Steinacker JM. Physiological aspects of training in rowing. Int J Sports Med 1993;14 Suppl 1:S3-10.

2. Mazzone T. Kinesiology of the rowing stroke. NSCA Journal 1988;10:4-11.

3. Rodriguez RJ, Rogriguez RP, Cook SD, Sandborn PM. Electromyographic analysis of rowing stroke biomechanics. J Sports Med Phys Fitness 1990;30:103-8.

4. Baudouin A, Hawkins D. Investigation of biomechanical factors affecting rowing performance. J Biomech 2004;37:969-76.

5. Hawkins D. A new instrumentation system for training rowers. J Biomech 2000;33:241-5.

6. Smith RM, Loschner C. Biomechanics feedback for rowing. J Sports Sci 2002;20:783-91.

7. Page $P$, Hawkins D. A real-time biomechanical feedback system for training in rowers. Sports Engineering. 2003;6:67-80.

8. Hill H. Dynamics of coordination within elite rowing crews: evidence from force pattern analysis. J Sports Sci 2002;20:101-17.

9. Roth W, Schwanitz P, Pas P, Bauer P. Force-time characteristics of the rowing stroke and corresponding physiological muscle adaptations. Int J Sports Med 1993;14 Suppl 1:S32-4.
10. Cheng B, Kuipers $H$, Snyder AC, Keizer HA, Jeukendrup A, Hesselink M. A new approach for the determination of ventilatory and lactate thresholds. Int J Sports Med 1992;13:518-22.

11. Baptista R, Oliveira L, Figueiredo G, Contieri J, Loss J, Oliveira A. Limiar de lactato em remadores: comparação entre dois métodos de determinação. Rev Bras Med Esporte 2005;11(4).

12. Ingham SA, Whyte GP, Jones $K$, Nevill AM. Determinants of 2,000 m rowing ergometer performance in elite rowers. Eur J Appl Physiol 2002;88:243-6.

13. Riechman SE, Zoeller RF, Balasekaran G, Goss FL, Robertson RJ. Prediction of $2000 \mathrm{~m}$ indoor rowing performance using a 30 s sprint and maximal oxygen uptake. J Sports Sci 2002;20:681-7.

14. Bourdin M, Messonnier L, Hager JP, Lacour JR. Peak power output predicts rowing ergometer performance in elite male rowers. Int J Sports Med 2004;25:368-73.

15. Favero TG, Zable AC, Colter D, Abramson JJ. Lactate inhibits Ca(2+)-activated $\mathrm{Ca}(2+)$-channel activity from skeletal muscle sarcoplasmic reticulum. J Appl Physiol 1997;82:447-52.

16. Lucia A, Sanchez O, Carvajal A, Chicharro JL. Analysis of the aerobic-anaerobic transition in elite cyclists during incremental exercise with the use of electromyography. Br J Sports Med 1999;33:178-85. 